\title{
Wearable Sensors for Pervasive Healthcare Management
}

\author{
Daniel Olguín Olguín \\ Human Dynamics Group \\ MIT Media Laboratory \\ Cambridge, MA 02139 \\ dolguin@media.mit.edu
}

\author{
Peter A. Gloor \\ Center for Collective Intelligence \\ MIT Sloan School of Management \\ Cambridge, MA 02139 \\ pgloor@mit.edu
}

\author{
Alex (Sandy) Pentland \\ Human Dynamics Group \\ MIT Media Laboratory \\ Cambridge, MA 02139 \\ pentland@media.mit.edu
}

\begin{abstract}
We show that it is possible to identify individual personality traits and measure group performance in a Postanesthesia Care Unit (PACU) using wearable sensors. We instrumented a group of 67 nurses working in the PACU of a Boston area hospital with sociometric badges capable of measuring physical activity, speech activity, face-to-face interaction, and physical proximity. Using the data collected with these sensors we were able to estimate the daily average length of stay (LOS) and number of delays.
\end{abstract}

\section{INTRODUCTION}

Combining wearable technology with pervasive computing devices, such as sensors or actuators, is an approach with high potential for being an important part of IT solutions for applications in hospital environments [1].

Our research group has developed several wearable sensing platforms to automatically capture individual and collective patterns of behavior, predict human behavior from unconscious social signals, identify social affinity among individuals working in the same team, and enhance social interactions by providing real-time feedback.

Post-anesthesia care unit (PACU) monitoring reduces morbidity and is the standard of care for post-surgical patients. PACUs require large nurse to patient ratios, which contributes to the cost of care [2]. Reducing the patient's length of stay (LOS) within the PACU can control or even lower costs. The length of time a patient remains in the PACU is medically attributed to the anesthetic drugs used during the operation and additional side effects that may occur, such as nausea and vomiting [3]. Previous studies demonstrate that as many as $20 \%$ of patients experience delayed discharge from the PACU and over half of the delays may be personnel-related or due to personnel shortages and inefficiencies [4].

We instrumented a group of 67 nurses working in the Post Anesthesia Care Unit (PACU) of a Boston area hospital with sociometric badges capable of measuring physical activity, speech activity, face-to-face interaction, and physical proximity. Using the data collected with these sensors we have been able to identify different personality traits and estimate the daily average length of stay (LOS) and number of delays.

\section{BACKGROUND}

One of the first attempts to measure face-to-face interactions between people using wearable sensors was the sociometer [5].
This wearable sensor package was used to learn social interactions from sensory data and model the structure and dynamics of social networks [6]. Pentland describes several statistical learning methods that use wearable sensor data to make reliable estimates of users' interactions [7]. He presents a detailed description of eigenbehavior modeling for learning and classifying user behavior from proximity and location data, and influence modeling for predicting the behavior of a subject from another subject's data. Our latest research platform uses sociometric badges to measure and analyze organizational behavior [8].

\section{A. Personality}

Over time researchers have tried to describe and measure personality traits (individual tendencies to react emotionally or behaviorally in a specific way) using various tests. The most popular model is the "Big Five" model that describes five personality traits [9]: Neuroticism $(\mathrm{N})$, extroversion (E), openness (O), Agreeableness (A), and conscientiousness (C).

\section{B. Group Behavior}

The study of groups has been a focus across the social and behavioral sciences for over 50 years. Poole et al. have described nine different interdisciplinary perspectives on small groups [10]. We are particularly interested in the socialevolutionary perspective which posits that group structure and interaction reflect evolutionary forces that have shaped human social behaviors over thousands of years. This perspective treats groups as aggregates of individuals and views group behavior as the product of individual behaviors that scale up to the group level.

\section{METHOD}

\section{A. Participants}

The study sample was composed of 67 nurses who worked in the PACU of a Boston-area hospital. Each nurse wore a sociometric badge every day for a period of 27 days. In total we collected 3,906 hours of data. The mean number of hours each participant wore a badge was 7.18 hours per day $( \pm 4.17)$. During this period a total of 1128 patients were admitted to the PACU, with an average LOS of $235.66( \pm 261.76)$ minutes. 
At the end of the study participants answered a NEO-FFI (NEO Five Factor Inventory) questionnaire [11] that contains 60 questions and is designed to measure the five personality traits described in the background section.

\section{B. Experimental set-up}

The hospital has 50 Operation Rooms (OR). After surgery is completed, patients are taken to the PACU, where they are kept under supervision until they recover from anesthesia. Thereafter they are admitted to the floor units where they convalesce before being discharged. Patients without assigned beds on the floors are kept in the PACU until vacancies on the floors can be found. The PACU is a critical intermediary step in the surgical patient throughput system and it consistently experiences delays of various kinds. These delays cause hold ups in the OR resulting in schedule disruptions, overtime work and productivity losses. This translates into loss of revenue for the hospital since the health-care system reimburses a fixed sum for a particular surgical procedure irrespective of the patient's length of stay in the hospital [12].

We placed base stations next to each bed and phone in the PACU in order to detect when the nurses were in close proximity to a bed or a phone and track their location and displacement patterns. There were 37 beds in the PACU, with only 30 being used during the study and 12 phones distributed around the room.

The sociometric badges and base stations broadcasted their ID every 5 seconds using a $2.4 \mathrm{GHz}$ transceiver $\left(T R_{\text {radio }}=12\right.$ transmissions per minute) and each time another badge or base station received a radio packet it logged the sender's ID and the radio signal strength. This allowed us to track the location of the nurses and detect when they were in close proximity to other nurses, beds or phones. The badges also transmitted their ID every 2 seconds using an infrared (IR) transceiver $\left(T R_{i r}=30\right.$ transmissions per minute).

\section{Procedure}

The daily average and standard deviation of the badge features described in the measurements section were calculated for each participant. We used correlation analysis to identify personality traits from the individual daily features, and stepwise multiple linear regression analysis to predict the daily average LOS and number of delays from the daily features aggregated across subjects.

\section{Measurements}

The sociometric badges shown in figure 1 are capable of measuring physical activity, speech activity, face-to-face interaction, and physical proximity.

\section{A. Physical activity}

A 3-axis accelerometer signal sampled at $f_{s} \geq 30 \mathrm{~Hz}$ captures the full range of human movement since $99 \%$ of the acceleration power during daily human activities is contained below $15 \mathrm{~Hz}$ [13]. The acceleration signal vector magnitude $\left(\left|\vec{a}_{i}^{\prime}\right|\right)$ provides a measure of the degree of movement intensity

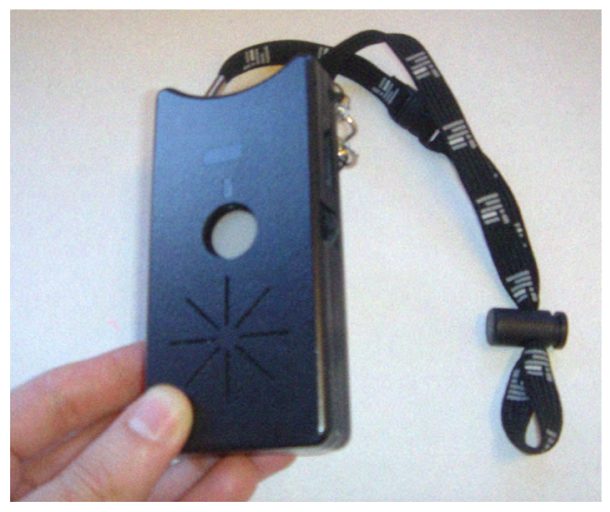

Fig. 1. Wearable sociometric badge

that includes the effect of signal variations in the three axes of acceleration [14]. From the 3-axis accelerometer signal we calculate the following features every minute:

- $\left(F^{1}\right)$ Mean accelerometer signal magnitude

- $\left(F^{2}\right)$ Standard deviation of signal magnitude

- $\left(F^{3}\right)$ Power or signal energy

\section{B. Speech activity}

Several speech enhancement and speech recognition frontend systems based on band-pass filter banks have been shown to be effective in detecting speech [15], [16]. The sociometric badges have an analog band-pass filter bank that divides the speech frequency spectrum $[85,4000] \mathrm{Hz}$ into four frequency bands: $f_{1}$ from 85 to $222 \mathrm{~Hz}, f_{2}$ from 222 to $583 \mathrm{~Hz}, f_{3}$ from 583 to $1527 \mathrm{~Hz}$, and $f_{4}$ from 1527 to $4000 \mathrm{~Hz}$. We compute the speech volume modulation from the output of filter 1 , since that is where the majority of the speaking energy resides. The amount of speaking time per minute is simply calculated by counting the number of samples in one minute where the volume modulation is $v(i)>0$. The band-pass filters also allow us to detect voiced and unvoiced speech and calculate the voiced speaking time. The following features are calculated from the filtered speech signal every minute:

- $\left(F^{4}\right)$ Mean volume modulation

- $\left(F^{5}\right)$ Standard deviation of volume modulation

- $\left(F^{6}\right)$ Speaking time

- $\left(F^{7}\right)$ Voiced speaking time

\section{Face-to-face interaction ( $f 2 f)$}

IR transmissions can be used as a proxy for the detection of face-to-face interaction between people [5]. In order for one badge to be detected through IR, two sociometric badges must have a direct line of sight and the receiving badge's IR sensor must be within the transmitting badge's IR signal cone of height $h \leq 1$ meter and radius $r \leq h \tan \theta$, where $\theta= \pm 15^{\circ}$. We define the amount of face-to-face interaction as the total number of IR detections per minute divided by the IR transmission rate $\left(T R_{i r}\right)$. Using the IR detections we calculate the following features every minute:

- $\left(F^{8}\right)$ Face-to-face interaction time

- $\left(F^{9}\right)$ Number of different people with f2f interaction 


\section{Proximity}

RSSI (radio signal strength indicator) is a measure of the signal strength between transmitting and receiving devices. An average threshold was determined experimentally in order to detect when two badges were in close proximity to each other (at a distance of less than 3 meters) by collecting RSSI measurements over an extended period of time under different environmental conditions. The time spent in close proximity to another person, a bed, and a phone are calculated by dividing the number of radio packets with $\mathrm{RSSI}>\mathrm{RSSI}_{t h}$ by the radio transmission rate $\left(T R_{\text {radio }}\right)$. Using the proximity detections we calculate the following features every minute:

- $\left(F^{10}\right)$ Time in close proximity to other people

- $\left(F^{11}\right)$ Time in close proximity to a bed

- $\left(F^{12}\right)$ Time in close proximity to a phone

Table I shows the notation that we use when we refer to the daily features.

\section{RESULTS}

We will use the following notation to distinguish between daily features calculated across days and daily features calculated across subjects:

$\mu\left(F^{n}\right)_{D}$ denotes the average of daily feature $F^{n}$ across days.

$\sigma\left(F^{n}\right)_{D}$ denotes the standard deviation of daily feature $F^{n}$ across days.

$\mu\left(F^{n}\right)_{S}$ denotes the average of daily feature $F^{n}$ across subjects.

$\sigma\left(F^{n}\right)_{S}$ denotes the standard deviation of daily feature $F^{n}$ across subjects.

Table II shows the correlation coefficients between the daily badge features (mean and standard deviation across days) from each participant's sensor data and the results of their personality test grouped by behavior description. These results do not imply causality and can be interpreted as follows:

- Neuroticism. The higher the daily percentage of f2f time, and the more variation across days in the daily percentage of $\mathrm{f} 2 \mathrm{f}$ time, the more neurotic.

- Extroversion. The lower the daily average time in close proximity to a bed or phone, the lower the daily variation in time in close proximity to a phone (phone call length), and the less variation across days in the daily variation in time in close proximity to a phone, the more extrovert.

- Openness. The higher the daily variation in physical activity, the less variation across days in daily variation in speaking time, and the more variation across days in the daily average time in close proximity to a phone, the more open.

- Agreeability. The less variation across days in the daily average speech volume modulation, the less variation across days in the daily variation in speech volume modulation, and the less variation across days in the daily percentage of time in close proximity to a bed, the more agreeable.
TABLE III

PREDICTION OF DAILY AVERAGE LOS IN MINUTES FROM BADGE FEATURES ACROSS SUBJECTS $(p<0.005)$

\begin{tabular}{lrrrrr}
\hline Predictors & $R$ & $R^{2}$ & $F$ & RMSE & $\beta$ \\
\hline$\sigma\left(F_{\mu}^{1}\right)_{S}$ & -0.72 & 0.52 & 18.11 & 36.32 & -1387.52 \\
$\sigma\left(F_{\%}^{8}\right)_{S}$ & 0.53 & 0.71 & 19.39 & 29.07 & 2909.17 \\
$\sigma\left(F_{\%}^{12}\right)_{S}$ & 0.16 & 0.79 & 19.21 & 25.24 & -53.5 \\
\hline
\end{tabular}

TABLE IV

PREDICTION OF DAILY AVERAGE NUMBER OF DELAYS (GOING OUT OF THE PACU) FROM BADGE FEATURES ACROSS SUBJECTS $(p<0.05)$

\begin{tabular}{lrrrrr}
\hline Predictors & $R$ & $R^{2}$ & $F$ & RMSE & $\beta$ \\
\hline$\sigma\left(F_{\mu}^{2}\right)_{S}$ & 0.46 & 0.21 & 4.48 & 3.95 & -189.53 \\
$\sigma\left(F_{\mu}^{12}\right)_{S}$ & -0.60 & 0.56 & 10.37 & 3.02 & 206.63 \\
\hline
\end{tabular}

Tables III and IV show that it is possible to explain the variation in the daily average LOS in minutes $\left(R^{2}=0.79\right)$ and the daily average number of outgoing delays $\left(R^{2}=0.56\right)$ from the aggregated features across subjects. In the case of LOS, the variation in physical activity intensity, face-to-face interaction time, and time in close proximity to a phone across subjects played an important role. Low variation across the nurses' level of physical activity (either all nurses having high levels of activity or low levels of activity) and high variation across the nurses' face-to-face interaction time was an indication of extended LOS. In the context of the PACU these results can be interpreted as either most PACU nurses being busy (high activity levels) or waiting for bed availability (low activity levels). The variation across the nurses' face-to-face interaction time could be an indicator of poor communication among nurses. The variation in the detection of nurses in close proximity to a phone could be an indicator of lack of advanced notification to the receiving unit of an impending patient transfer.

When estimating the daily number of delays the variation across subjects in their individual physical activity variation throughout the day and the average time they are in close proximity to a phone (which could be related to phone call length) were the most predictive features. This means that a high variation across the nurses' daily activity levels (having alternate periods of high activity and low activity during the day), coupled with the variation in the time they spend in close proximity to a phone, is an indication of increased number of delays in the PACU.

\section{CONCLUSIONS}

The use of pervasive technology in healthcare management has the potential to improve organizational performance by allowing healthcare providers to identify bottlenecks and ineffective behaviors. We presented experimental results that show that it is possible to identify individual personality traits and measure group performance (reflected in the average LOS and number of delays) from low-level sensor data. This is a first attempt to measure and model organizational performance at the individual and group levels. 
TABLE I

DAILY SENSOR FEATURES, WHERE $h(k)=1$ IF $F^{1}(k)>1$ (WHEN WEARING THE BADGE), AND $h(k)=0$ IF $F^{1}(k) \leq 1$ (WHEN NOT WEARING THE BADGE)

\begin{tabular}{|l|l|l|}
\hline Daily feature & Notation & Calculation \\
\hline Average & $F_{\mu}^{n}$ & $\frac{1}{\sum_{k=1}^{K} h(k)} \sum_{k=1}^{K} F^{n}(k) h(k)$ \\
\hline Standard deviation & $F_{\sigma}^{n}$ & $\sqrt{\frac{1}{\sum_{k=1}^{K} h(k)} \sum_{k=1}^{K}\left[F^{n}(k) h(k)-F_{\mu}^{n}(k)\right]^{2}}$ \\
\hline Percentage of time & $F_{\%}^{n}$ & $\frac{1}{\sum_{k=1}^{K} h(k)} \sum_{k=1}^{K} F^{n}(k)>0$ \\
\hline
\end{tabular}

TABLE II

CORRELATION COEFFICIENTS BETWEEN MONTHLY BADGE FEATURES AND PERSONALITY TRAITS. ${ }^{*} p<0.05,{ }^{* *} p<0.01$.

\begin{tabular}{llccccc}
\hline & & \multicolumn{5}{c}{ Personality Dimension } \\
\cline { 3 - 7 } Behavior Description & Feature & $\mathrm{N}$ & $\mathrm{E}$ & $\mathrm{O}$ & $\mathrm{A}$ & $\mathrm{C}$ \\
\hline Physical activity & $\mu\left(F_{\sigma}^{2}\right)_{D}$ & -0.03 & -0.07 & $0.37^{*}$ & -0.14 & -0.09 \\
\hline Speech activity & $\sigma\left(F_{\mu}^{5}\right)_{D}$ & 0.07 & -0.16 & -0.18 & $-0.43^{* *}$ & -0.006 \\
& $\sigma\left(F_{\sigma}^{5}\right)_{D}$ & 0.09 & -0.13 & -0.24 & $-0.41^{* *}$ & 0.12 \\
& $\sigma\left(F_{\sigma}^{6}\right)_{D}$ & 0.11 & -0.006 & $-0.36^{*}$ & -0.18 & 0.02 \\
\hline Face-to-face & $\mu\left(F_{\%}^{8}\right)_{D}$ & $0.35^{*}$ & -0.08 & 0.004 & 0.06 & -0.18 \\
& $\sigma\left(F_{\%}^{8}\right)_{D}$ & $0.41^{*}$ & -0.09 & 0.02 & 0.06 & -0.26 \\
\hline Proximity & $\sigma\left(F_{\%}^{11}\right)_{D}$ & 0.06 & -0.18 & -0.16 & $-0.34 *$ & 0.16 \\
& $\mu\left(F_{\mu}^{11}\right)_{D}$ & -0.003 & $-0.36^{*}$ & 0.11 & -0.26 & 0.18 \\
& $\mu\left(F_{\mu}^{12}\right)_{D}$ & 0.15 & $-0.39^{*}$ & 0.22 & -0.24 & -0.11 \\
& $\sigma\left(F_{\mu}^{12}\right)_{D}$ & 0.25 & -0.27 & $0.32^{*}$ & -0.18 & -0.20 \\
& $\mu\left(F_{\sigma}^{12}\right)_{D}$ & 0.12 & $-0.36^{*}$ & 0.22 & -0.20 & -0.14 \\
& $\sigma\left(F_{\sigma}^{12}\right)_{D}$ & 0.31 & $-0.34^{*}$ & 0.17 & -0.31 & -0.08 \\
\hline
\end{tabular}

Our results argue in favor of using wearable sensors to study group behavior, and incorporating behavioral data into patient scheduling systems to reduce the patient's LOS and save costs by minimizing the number of delays. We plan to extend this research to multiple groups and entire organizations. Future work includes modeling, simulation and optimization of individual and group behavior from sensor data.

\section{ACKNOWLEDGMENT}

The authors would like to thank Daniel Dolan, Warren Sandberg, Scott Ciesielski, Chandrika Samarth, Miki Hayakawa and the nurses who participated in the study for their contribution to this project.

\section{REFERENCES}

[1] V. Carlsson, T. Klug, T. Ziegert, and A. Zinnen, "Wearable computers in clinical ward rounds," in Proceedings of the 3rd International Forum on Applied Wearable Computing, Bremen, Germany, Mar 2006, pp. 1-9.

[2] J. I. Waddle, A. S. Evers, and J. F. Piccirillo, "Postanesthesia care unit length of stay: Quantifying and assessing dependent factors," Anesthesia \& Analgesia, vol. 87, pp. 628-633, 1998.

[3] T. W. Zollinger and R. M. J. Saywell, "Delays in patient transfer: Postanesthesia care nursing," Nursing Economics, 1999.

[4] M. A. Meyer, "Use of location data for the surveillance, analysis, and optimization of clinical processes," Master's thesis, Massachusetts Institute of Technology, June 2006.

[5] T. Choudhury and A. Pentland, "Sensing and modeling human networks using the sociometer," in Proceedings of the 7th IEEE International Symposium on Wearable Computers, October 2003, pp. 216-222.

[6] T. Choudhury, "Sensing and modeling human networks," Ph.D. dissertation, MIT Media Laboratory, Feb 2004.
[7] A. Pentland, "Automatic mapping and modeling of human networks," Physica A, vol. 378, no. 1, pp. 59-67, May 2006.

[8] D. Olguin-Olguin, B. N. Waber, T. Kim, A. Mohan, K. Ara, and A. S. Pentland, "Sensible organizations: Technology and methodology for automatically measuring organizational behavior," To appear in IEEE Transactions on Systems, Man, and Cybernetics-Part B: Cybernetics, vol. 39, no. 1, 2009.

[9] H. L. Tosi, N. P. Mero, and J. R. Rizzo, Managing Organizational Behavior. Cambridge, MA: Blackwell Publishers Inc., 2000.

[10] M. S. Poole, A. B. Hollingshead, J. E. McGrath, R. L. Moreland, and J. Rohrbaugh, "Interdisciplinary perspectives on small groups," Small Group Research, vol. 35, no. 1, pp. 3-16, 2004.

[11] P. T. Costa and R. R. McCrae, "Neo five-factor inventory," 2008, psychological Assessment Resources, Inc. [http://www3.parinc.com/products/ product.aspx?Productid=NEO_FFI]. Accessed on: 09/18/2008.

[12] C. N. Samarth, "It adoption in hospitals: Social networking, governance and the clockspeed of change," Master's thesis, Massachusetts Institute of Technology, June 2007.

[13] M. J. Mathie, A. C. F. Coster, N. H. Lovell, and B. G. Celler, "Accelerometry: providing an integrated, practical method for long-term ambulatory monitoring of human movement," Physiological Measurement, vol. 25, no. 4, pp. R1-R20, 2004.

[14] D. M. Karantonis, M. R. Narayanan, M. Mathie, N. H. Lovell, and B. G. Celler, "Implementation of a real-time human movement classifier using a triaxial accelerometer for ambulatory monitoring," IEEE Transactions on Information Technology and Biomedicine, vol. 10, no. 1, pp. 156167, Jan 2006.

[15] R. Ellis, H. Yoo, D. W. Graham, P. Haseler, and D. V. Anderson, "A continuous-time speech enhancement front-end for microphone inputs," in Proceedings of the IEEE International Symposium on Circuits and Systems, May 2002.

[16] A. Mouchtaris, Y. Cao, S. Khan, J. V. der Spiegel, and P.Mueller, "Combined software/hardware implementation of a filterbank front-end for speech recognition," in Proceedings of the IEEE Workshop on Signal Processing Systems Design and Implementation, November 2005. 